\title{
Energy efficient service differentiated QoS aware routing in cluster-based wireless sensor network
}

\author{
Jayashree Agarkhed and \\ Yogita Dattatraya Patil ${ }^{\star}$
}

Computer Science and Engineering Department, Poojya Doddappa Appa (PDA) College of Engineering, Visvesvaraya Technological University (VTU), Kalaburagi 585101, Karnataka, India

Email: jayashreeptl@yahoo.com

Email: agyogita@gmail.com

*Corresponding author

\begin{abstract}
In today's technological era, WSNs has gained attention worldwide with its miniature size and low cost. Data transmission demands both energy and quality of service (QoS) to ensure efficient use of the sensors and adequate access to collecting information. Lot of research work in literature has been focused on QoS provisioning by differentiated service technique that differentiates and priorities different traffic classes to meet the user requirements. Our work toward scheduling based on packet type providing high priority to emergency data to facilitate reliable transmission of emergency data in critical situations. We proposed an approach for effective sensing by use of stochastic scheduling to increase the energy efficiency of sensor nodes for intracluster communication. The proposed technique in this work outperforms when compared with the existing protocol in the literature in terms of minimised energy consumption, delay and high throughput by offloading of the energy-intensive tasks.
\end{abstract}

Keywords: clustering; energy efficiency; proxy server; time division multiple access; IEEE 802.15.4; IEEE 802.15.6; differentiated service; hybrid; intelligence.

Reference to this paper should be made as follows: Agarkhed, J. and Patil, Y.D. (2019) 'Energy efficient service differentiated QoS aware routing in cluster-based wireless sensor network', Int. J. Hybrid Intelligence, Vol. 1, No. 1, pp.79-95.

Biographical notes: Jayashree Agarkhed is currently working as Professor in the Computer Science and Engineering Department at Poojya Doddappa Appa College of Engineering, Kalaburagi, affiliated to Visvesvaraya Technological University, Belagavi. She obtained her MTech in Computer Science and Engineering in 2003 from PDA College of Engineering and awarded with $\mathrm{PhD}$ degree in 2013 in Electrical Science from VTU. Her main research areas are in wireless sensor network, multimedia information networks, artificial intelligence, cloud computing, and internet of things. She has published more than 120 scientific articles in top-tier journals and conferences, including Springer journal, IEEE conference proceedings and Springer Book chapters. 
Yogita Dattatraya Patil is currently working as an Associate Professor in the Computer Science and Engineering Department at APPA Institute of Engineering and Technology, Kalaburagi and pursuing a $\mathrm{PhD}$ from Poojya Doddappa Appa (PDA) College of Engineering, Kalaburagi, affiliated to Visvesvaraya Technological University (VTU), Belagavi. She has obtained a BE from the Pune University and an MTech in Computer Science and Engineering in 2010 from VTU. Her area of interest includes wireless sensor network, multimedia communication, cloud computing and the internet of things.

\section{Introduction}

Wireless sensor networks (WSNs) consist of a large number of configurable sensors, to meet the requirements of various applications. The node in WSNs is a low power device that includes one or more sensors, storage unit, a processing unit, power source, radio and actuator. Various types of sensors vary slightly regarding functionality provided by them. A sensor node may use a mechanical, biological, chemical or magnetic sensor to meet the requirements of industry, military applications, precision agriculture applications and healthcare monitoring applications with ease of implementation and maintenance cost. Sensor nodes have two-fold capabilities (Alam et al., 2015). Primarily, they are capable to sense variety of environmental conditions such as pressure, sound level, temperature, humidity variations, vibrations, etc. Secondly they also have computing capability. Multi-hop communication establishes a wireless connection among nodes, facilitates fast transmission of sensed data from a source node to the respective destination or specific master node (Patil and Agarkhed, 2015). It is challenging to sustain massive operation on sensor nodes for extended running time since sensors operate on a battery. It is also very difficult to recharge or change batteries for the sensor nodes.

Its use for various smart applications has motivated researchers to device new protocol for the development of smart building, a smart cities, smart healthcare centre, etc. With advancement in technology, sensor nodes incorporate intelligence and support to build an internet of things (IoT) (Tjensvold, 2007).

Sensor node poses many constraints like limited amount battery power and difficulty of its recharging if used in harsh environment, limited storage capability, dealing with interoperability problems, etc. Sensor nodes low power, low processing capability, demands energy conservation by avoiding unnecessary sensing activities (Luo et al., 2010; Yun et al., 2010; Wang et al., 2003; Bai et al., 2009; Zhou et al., 2004) by the development of proper scheduling methods (Liu et al., 2006a; Patil et al., 2015). Another biggest challenge in WSNs is the efficient design of routing protocol because of several network constraints such as random node deployment, energy consumption, data delivery models, node capability, network dynamic and data aggregation. One such issue of limited energy source overcome by use of a genetic algorithm for continuous monitoring of target (Elhoseny et al., 2018).

Limited storage capability, one of the constraints of WSN can be overcome by integrating WSNs with cloud computing, which not only provides storage as a service but also supports computation as a service (Patil and Agarkhed, 2016). Computing resources like memory, processor, and storage accessed at any user location by use of virtualisation 
techniques of cloud computing (Armbrust et al., 2009). Cloud computing can, in turn, facilitate abundant storage and additional computing resources to the sensor node. This demands satisfying user/application specific requirements with resource constraint WSN. Quality of service (QoS) support needs tuning parameters at medium access layer (MAC) to support medium sharing and reliable communication, achieved by clustering of nodes and controlling their duty cycle. Another mechanism to meet application requirements is by use of service differentiation to differentiate and prioritise traffic flow. The study of literature motivates to design energy efficient service differentiated QoS aware routing in cluster-based (EESDRCB) WSN to achieve maximum throughput with negligible delay.

In this paper, we have proposed a cluster-based routing technique which minimises energy consumption of sensor nodes by scheduling based on predicted sensing error that in turn provide data accuracy. Reliable data transmission achieved by use of priority queues to offer differentiated service where each queue used for storing different types of data packets which in turn minimise delay and use the network bandwidth efficiently.

The rest of the paper is structured with the following sections. Section 2 details about related work done. Section 3 details about energy conservation by use of duty cycle scheduled method and offloading the computations. Section 4 discusses proposed technique. Section 5 highlights on the evaluation of performance. Section 6 concludes with the need for EESDRCB.

\section{Related works}

One of the goals of routing in WSN is to conserve energy. Therefore, energy efficient routing protocols are needed in sensor network as the network lifetime depends on the battery life of the nodes. One basic protocol low energy adaptive clustering hierarchy (LEACH) (Heinzelman et al., 2002) allows cluster heads (CHs) to directly communicate with sink. A cluster formed by set of sensor node based on the received signal strength of node and use $\mathrm{CH}$ as a router to sink with the constraint that maximum of $5 \%$ of nodes can be $\mathrm{CH}$ in a network. LEACH has less energy consumption than direct communication by establishing a distributed network without global knowledge of the network. LEACH has lost its importance as it cannot be used for large size network where selecting $\mathrm{CH}$ is difficult. LEACH-F has fixed number of clusters and $\mathrm{CH}$ is rotated within its clusters. This may or may not support energy saving and not flexible for a mobile node. As LEACH use single hop communication leads to increase the distance between $\mathrm{CH}$ and sink, which in turn increase communication cost and shortens the lifetime of the network. Communication distance among nodes minimised by dividing a network according to an optimal number of clusters as specified in an optimal number of clusters algorithm (ONCA) (Jian et al., 2014).

Power efficient gathering in sensor information system (PEGASIS) use multi-hop routing by forming chains of sensor nodes and selects only one node to transmit to the base station (BS) instead of multiple nodes as in LEACH. It outperforms than LEACH by aggregating data from nodes. One drawback of PEGASIS is increased delay due to chain formation that is overcome by hierarchical PEGASIS. It is an extension of PEGASIS. It uses simultaneous transmission of data messages which may lead to collision and avoid by use of signal coding. The parallel data transmission can be achieved by chain-based 
protocol with CDMA capable nodes to reduce delay and needs dynamic topology adjustment since sensor energy is not tracked (Lindsey and Raghavendra, 2002).

Threshold sensitive energy efficient sensor network (TEEN) is a hierarchical protocol that responds to a sudden change in sensed attributes such as temperature and useful in time-critical applications using the network that operate in reactive mode. In this closer nodes, form cluster and this process go from the second level until-BS reaches. It uses either soft or hard threshold to trigger the sensor node to switch its transmitter. By varying these thresholds, data transmission can be controlled. TEEN is not useful for periodic reporting applications (Manjeshwar and Agrawal, 2001).

Adaptive threshold sensitive energy efficient sensor network (APTEEN) protocol capture periodic data and react to time-critical events same as TEEN. The performance of TEEN is best than LEACH and APTEEN (Manjeshwar and Agrawal, 2002).

Hybrid energy-efficient distributed (HEED) protocol is useful in ad-hoc sensor networks. HEED assumes all nodes initially have the same energy and further, all nodes chose themselves as a $\mathrm{CH}$ according to their remaining power and based on node degree for cluster election. HEED is a cluster-based multi-hop routing protocol. HEED achieves load balancing and uniform $\mathrm{CH}$ distribution. HEED does not have balanced energy consumption due generation of more CH (Younis and Fahmy, 2004).

Some of the above routing protocol discussed use single-path routing, where each sensor node routes its data through the shortest path to the sink. The single path routing may cause path break up due to the failure of nodes and demands new route discovery. This increases energy consumption and failure of a node that causes dropping of packets and increased delay in transmission to the sink. In such case, it is desirable to avoid node failure by choosing alternative path thus forming multi-path routing that increases the number of possible routes providing the robust transmission and increased throughput. Also routing using single path does not provide enough bandwidth for data transmission as WSN has limited bandwidth capability. If multiple paths are chosen for simultaneous transmission, then the aggregated bandwidth of the paths can meet actual the bandwidth requirement of any particular application. Increase in bandwidth minimises overall delay by use of multiple paths providing load balancing energy utilisation among the nodes, in turn, increasing network lifetime (Jayashree et al., 2012; Agrakhed et al., 2012).

Kwak et al. (2010) discussed WSN support for real-time health monitoring of a long-suffering, patient and diagnoses of various life-threatening diseases. Continuous health monitoring conserves a lot of energy in sensing many health parameters using different types of sensor like temperature, pulse, breath, ECG sensors, etc.

A useful way to save up energy is to schedule data transmission, which in turn, reduces sensing power and preserve sensed data quality. One such algorithm for sensing and scheduling sensor nodes called CIES share sensing error information and controls sensing errors through neighbour node coordination achieving high data accuracy and throughput (Patil et al., 2015; Patil and Agarkhed, 2016; Kwak et al., 2010; Zhang et al., 2014). Further clustering helps to group sensor nodes into the cluster to accomplish the network scalability, to achieve load balancing, to reduce the size of the routing table at individual nodes (Akkaya and Younis, 2005), to save up communication bandwidth by using inter-cluster interaction to CHs (Younis et al., 2002). To conserve energy, CH can schedule activities in the cluster by switching the node in active and sleep state (Adamou et al., 2001; Wu and Biswas, 2005). Elhoseny et al. (2015) proposed a method to optimise exhaustion of sensor node energy by clustering sensor nodes using a genetic algorithm to 
extend the network life. Yuan et al. (2017) considered each sensor node the residual energy, amount of energy used in sensing, nodes distance to reach the BS and the number of vicinity nodes to find an optimal, dynamic network structure. The wireless body area network has been used for patient health monitoring. IEEE 802.15.6 has been established for standardisation of WBAN. This motivates way to provide a communication standard for deployment of low-power on body/in-body nodes for medical or non-medical applications (Jolly and Younis, 2005). IEEE 802.15.6 standard defines three PHY layers, i.e., narrowband, ultra wide band and human body communication layer that are based on needs of the application. Shehab et al. (2018) has presented performance evaluation of IEEE 802.11 , IEEE 802.15 and IEEE 802.16 for better coverage area.

From the study of related work, one crucial aspect considered is, achieving QoS parameters like data accuracy, minimised delay and minimised energy consumption to improve the lifetime of a sensor node.

\section{Sensor network architecture}

Findings of the extensive literature survey motivate to achieve the following objectives:

- $\quad$ energy efficient routing by clustering of nodes

- $\quad$ improve QoS, providing data accuracy by scheduling sensor nodes

- minimise delay by use of prioritised queues

- efficient bandwidth utilisation by compression of data.

These objectives achieved by EESDRCB architecture that consists of three models, i.e., energy-efficient data collecting model, task assignment model, and storage model, as shown in Figure 1.

Figure 1 Architecture of EESDRCB WSN (see online version for colours)

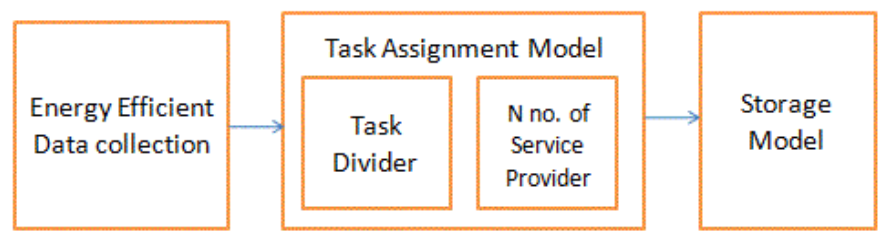

The EESDRCB architecture consists of three basic models. An energy efficient data collecting model concern with data sensing with accuracy and transmission of data to next model task assignment model, which separate the data stream as text and image to support differentiated service for efficient utilisation of bandwidth and to gain increased throughput. The third model is storage model that provide reliable storage of data.

\subsection{Energy efficient data collecting model}

Sensor nodes in the given sensing area are grouped into different clusters, considering their nearest distance to reach others. Here, the cluster-based approach for routing used 
and a node with maximum present energy selected as a $\mathrm{CH}$ and remaining nodes as cluster members. Thus in each cluster, a node with maximum remaining energy is elected as a $\mathrm{CH}$ as in Liu et al. (2006b). Each node support accurate data collection by use of a stochastic scheduling algorithm that employs the underlying data stream model and the data quality requirement to determine sampling instants for the sensor (Liu et al., 2005) and by use of sensing probability bound to control tolerable sensing error within each node (Zhang et al., 2008). The nodes within a cluster are scheduled to be active or in sleep mode based on sensing error estimation (Patil et al., 2015). The true value sensed differs from predicted value and leads to sensing error that can be caused by energy draining effect of each presently sensing sensor node. If the node subjected to sensing error beyond the set threshold, then that node is made to sleep and other nodes in the group are set to be in the active state to facilitate energy efficient transmission with the data accuracy. Intracluster communication is based on dynamic scheduling scheme. Each cluster member node checks data size or for the type of data stream and if the size of data is less than set threshold it performs compression and sends the data in compressed form to $\mathrm{CH}$. Each $\mathrm{CH}$ transmits data sensed by non-cluster members to the BS. Use of clustering, reduce transmission distance to sink as the transmission is through $\mathrm{CH}$. Inter-cluster communication is provided by inter-cluster communication, routing tree construction algorithm, based on the local energy consumption ratio of nodes (Yu et al., 2014).

\subsection{Task assignment model}

This model shown in Figure 1 consists of task divider (TD) and $N$ number of service providers (SPs). Once BS sends data to TD, it identifies the task, as a task with emergency data, task with compressed data and task with uncompressed data as shown in Figure 2. TD then uses the data flow, classifier as shown in Figure 3. The data flow classifier identifies the flow as emergency data flow, compressed data flow and uncompressed data flow and forwards them to respective queues. The queues accessed in round-robin fashion provided queue 1 with high priority for handling time-critical data.

- Queue 1 used to store emergency data packets and handled by the SP 1.

- Queue 2 used to store data packets compressed by sensor node and handled by the SP 2 .

- Queue 3 used to store data packets compressed by the proxy server and handled by the SP 3.

TD then sends the emergency data stored in queue 1 to SP 1 immediately. Compressed data in queue 2 are sent to SP 2. Uncompressed data is sent first to intermediate layer using a proxy server as shown in Figure 3. The proxy server, compress the data and submit to the SP 3. This avoids the collision of data traffic and increases throughput with zero delays for emergency data flow. The task of compressing large amount data offloaded to the proxy server. At the same time, TD can carry out the assignment of remaining tasks to the SP. Proxy server, compress large amounts of data independently and send compressed data to the SP 3. Multiple queues are used to support differentiated service by employing static priority assignment to data traffic. Each queue used for storing different types of data packets. 
Figure 2 Task classification (see online version for colours)

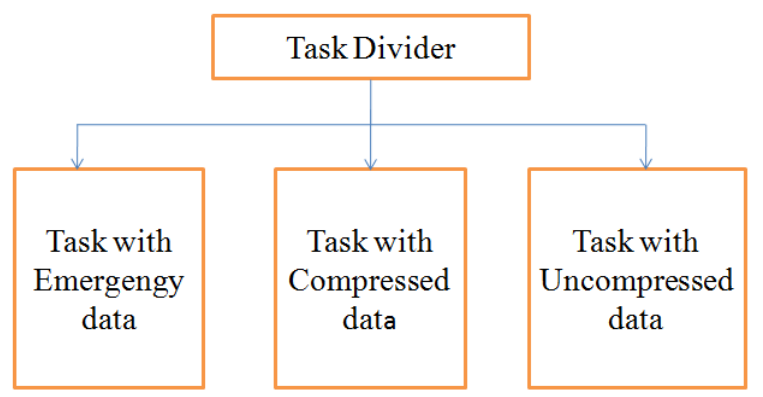

Figure 3 Data flow classifier (see online version for colours)

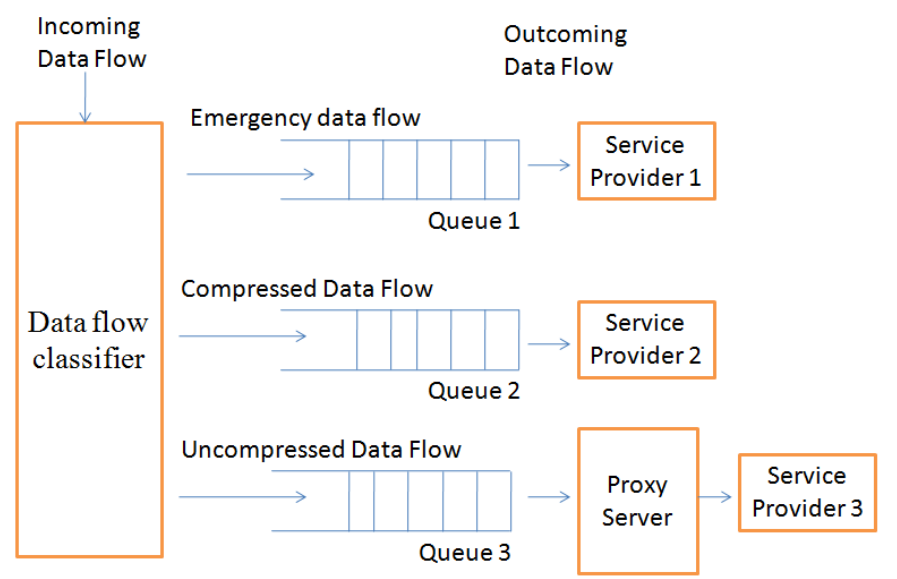

\subsection{Storage model}

Data arrival at SP is stored using cloud storage by employing multilevel security algorithm (Heinzelman et al., 2002). Since cloud service providers (CSP) work with third-party vendors, there is no guarantee that these vendors safeguard stored data in the cloud. For prevention of unauthorised access to cloud vendors, multilevel security algorithm uses three level security approaches. At first level, user password verification provides access to an authorised user. At second level, vendor password verification maintains vendor authentication and at third level security provided by encrypted key verification.

\section{Proposed techniques}

Energy efficient transmission of data, without compromising data accuracy and integrity is one of the biggest QoS requirements to be achieved in resource-constrained WSN. The proposed EESDRCB algorithm integrates sensing nodes to work in a collaborative way to save energy at each node by scheduling them to be in the active or sleep mode and 
avoiding failure of a network by selecting $\mathrm{CH}$ at each round with maximum remaining residual energy as discussed in Algorithm 1.

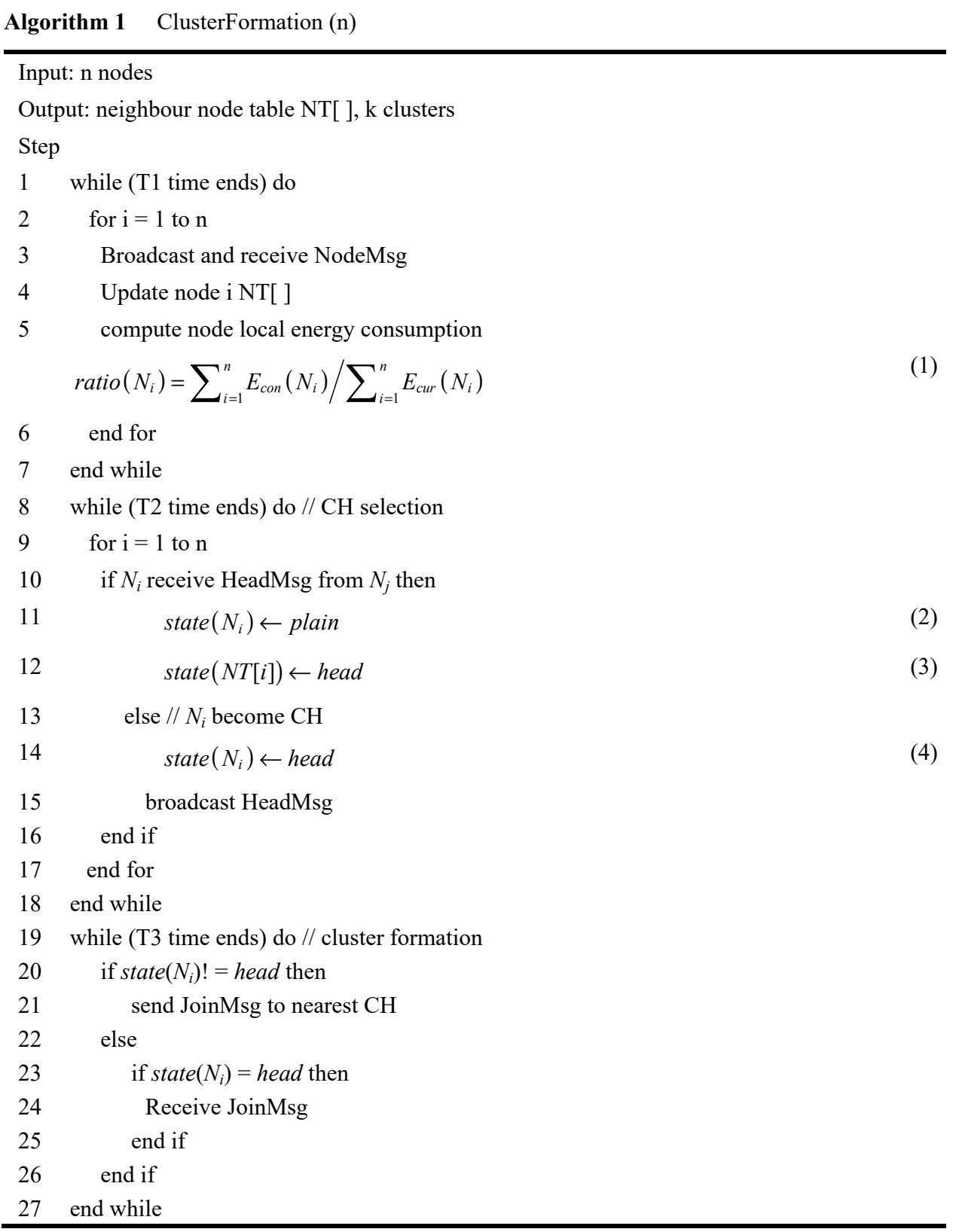


In cluster formation algorithm, each node broadcast NodeMsg with node id and current remaining energy. On receiving NodeMsg from neighbour node compute its local energy consumption ratio as per equation (1). All nodes complete this process in time T1. If $\mathrm{CH}$ has less local energy consumption ratio than other nodes, then it has a high chance of becoming a $\mathrm{CH}$. In cluster formation algorithm, $\mathrm{n}$ nodes send $\mathrm{n}$ NodeMsg, $\mathrm{n}-\mathrm{k}$ nodes (cluster members) send JoinMsg and $\mathrm{k}$ nodes send to HeadMsg. The overall control messages is $n+(n-k)+k=2 n$. Therefore, message control overhead complexity is $O(n)$. Time complexity is $\mathrm{O}(1)$ as loop runs for a constant node.

Algorithm 2 DataTransmission (k)

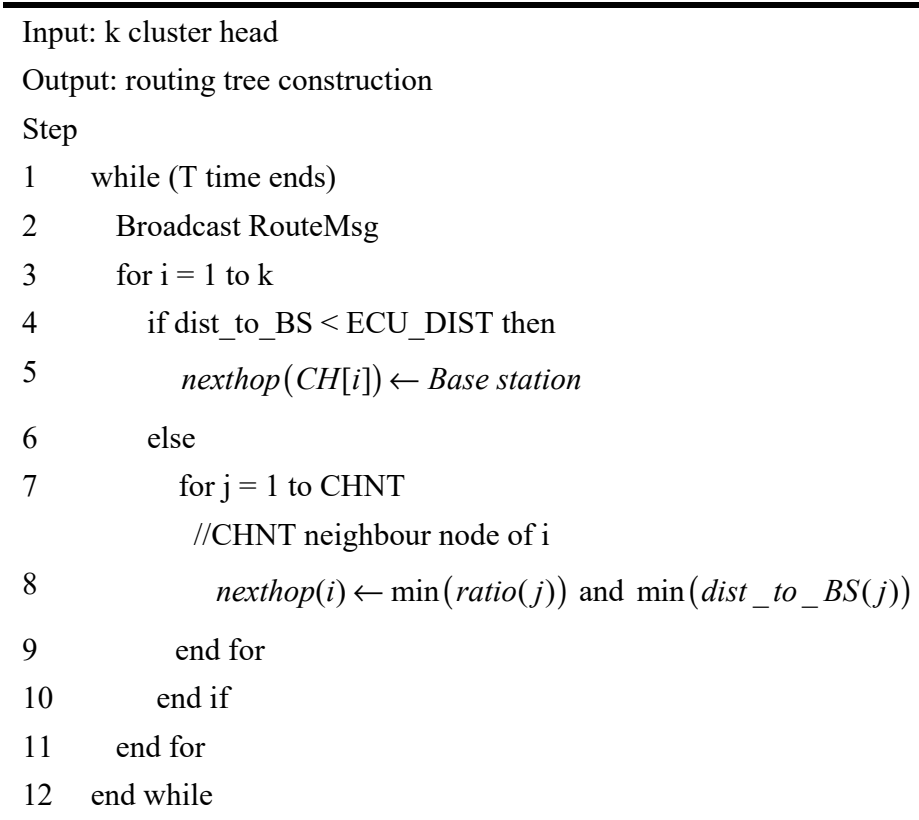

For intercluster communication, data transmission algorithm constructs routing tree among $\mathrm{CHs}$ within time duration $\mathrm{T}$. Each $\mathrm{CH}$ broadcast RouteMsg. Next, $\mathrm{CH}_{\mathrm{i}}$ chooses its next hop node as a BS (if its distance to BS is less than Euclidean distance) or as $\mathrm{CH}$ j that has a minimum distance to BS and minimum local energy consumption ratio among all its neighbour $\mathrm{CH}$ nodes. The running time complexity is where $\mathrm{m}=\mathrm{k}$ and $\mathrm{n}=\mathrm{CHNT}$ nodes.

The initialisation phase of EESDRCB specifies deployment of heterogeneous nodes in Step 1 forming $\mathrm{k}$ clusters. Nodes within the cluster are scheduled based on EPSEER and TDMA in Step 2. The data transmission, priorities emergency data flow and perform compression of data at sensor side for non-energy intensive task identified by Algorithm 4. For energy-intensive task, data transmitted to the proxy server further compressed. Data storage performed using multilevel security algorithm to provided authorised access. Running time complexity $=n+8(O(1))=O(n)$. 


\section{Algorithm 3 EESDRCB}

Input: $\mathrm{n}$ nodes

Output: k cluster heads, CHNT cluster head neighbour node table

Step

$1 \quad \mathrm{k} \leftarrow$ ClusterFormation (n) // cluster setup

2 schedule nodes within a cluster using EPSEER and TDMA

3 if dataflow $=$ emergency data then

4 Send data to service provider 1

5 else task $\leftarrow$ chk_energy_intensive(task)

6 if task NOT energy intensive

7 compress data at the sensor

$8 \quad$ else send data to the proxy server

9 compress data at the proxy

10 end if

11 end if

12 Call DataTransmission (k), send data to TD

13 Emergency data assignment to SP1

14 Compressed data assignment to SP2

15 Proxy compressed data assignment to SP3

16 Data storage using multilevel security algorithm

17 Performance evaluation

Algorithm 4 Chk_Energy_intensive (task)

Input: $T_{w}$ amount of computation, $S_{s d}$ sensor-equipped device speed, $S_{s}$ server speed, $d_{s}$ data sent, $B$ bandwidth, $P_{s d}$ sensor device power, $P_{t r}$ power required to send and receive data from a sensor over the network, $P_{\text {wait }}$ power consumed waiting for a result of the offloaded computation

Output: task status

Step

1 //Compute energy consumed at a sensor

$$
E_{s d}=P_{s d} \times \frac{T_{w}}{S_{s d}}
$$

where $E_{s d}$ : energy consumed

2 //Compute energy consumed at server

$$
E_{s}=P_{t r} \times \frac{d_{s}}{B}+P_{\text {wait }} \times \frac{T_{W}}{S_{s}}
$$

where $d_{s} / B$ : energy consumed to offload

3

$$
\text { If } E_{s d}>E_{s} \text { or } T_{w} \times\left(\frac{P_{s d}}{S_{s d}}-\frac{P_{i}}{S_{s}}\right)>P_{t r} \times \frac{d_{s}}{B}
$$

then

$$
\text { task=energy_intensive }
$$

4 Return task status 
Algorithm 4 takes into consideration the speed of a processor, speed of sensor device, bandwidth availability and input data to be exchanged, power needed to send this data and power consumed in waiting for results. In Step 1, compute the energy needed to execute task on sensor device in Step 2, energy consumed to execute a task on the server. Then set task as energy intensive if energy to execute the task at sensor side is more than at server side also considering data transmission from sensor to server is less as analysed in Step 3. Running time complexity is $\mathrm{O}(1)$.

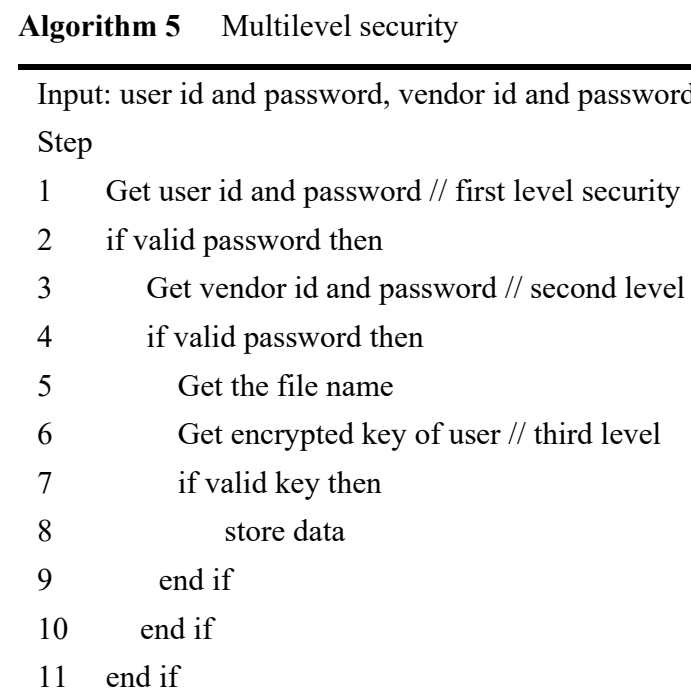

The multilevel security algorithm primarily checks for user authentication in Step 2, next for vendor authentication in Step 4 and finally, for encrypted key verification in Step 7 before performing data storage. Running time complexity is $\mathrm{O}(1)$.

Sensor node can use IEEE 802.15.4 or IEEE 802.15.6 standard. Using IEEE 802.15.6, average energy consumption and delay has been minimised with increased throughput as specified with simulation results in Section 5.

\section{Performance evaluations}

The performance of EESDCBR has been evaluated using NS2 simulation. The network using random deployment of nodes established in $1,000 \mathrm{~m} \times 500 \mathrm{~m}$ area. The BS is positioned at $50 \mathrm{~m}$ away from the specified deployment area containing 120 nodes forming 15 clusters. Figure 4 shows the simulation scenario comprising two-dimensional grid topology with 100 nodes along with TD, a SP, proxy server and storage. The channel capacity set to the 3e6 for each node. IEEE 802.15.4 and IEEE 802.15.6 have been used as MAC layer protocol. 
Figure 4 Simulation scenario with 120 nodes (see online version for colours)

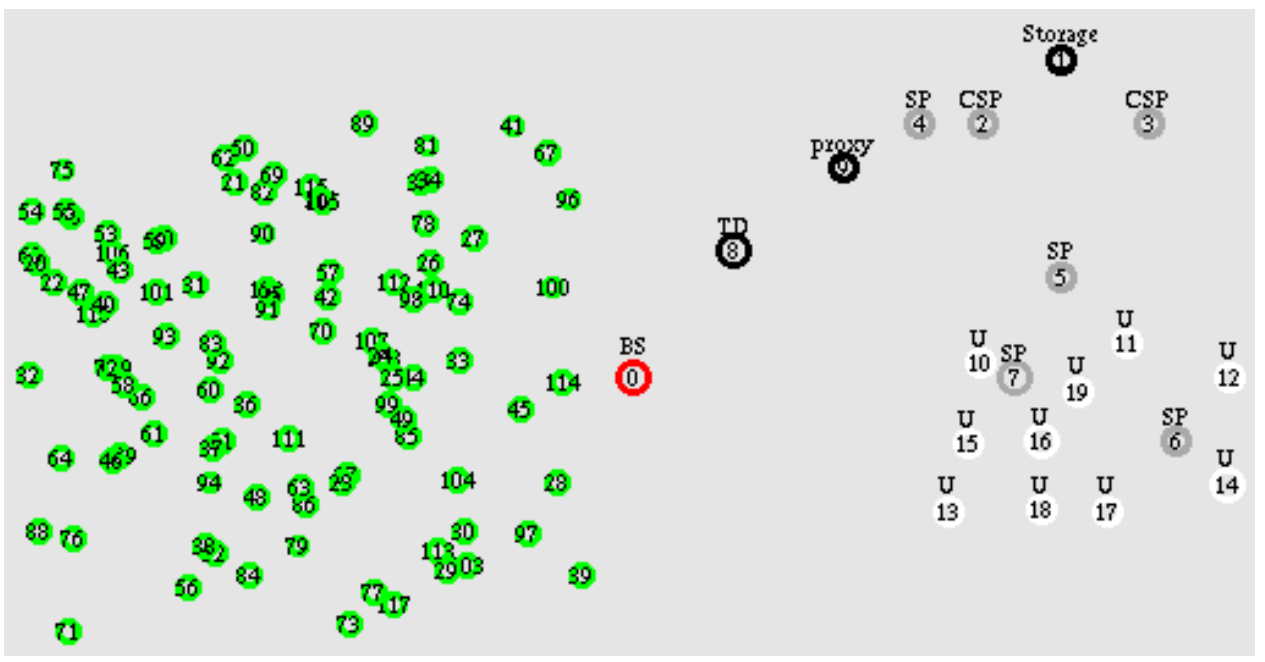

\subsection{Simulation parameters}

The effectiveness of proposed technique has been evaluated using NS2 simulations, with parameters listed in Table 1.

Table 1 Simulation parameters

\begin{tabular}{lc}
\hline Parameter & Value \\
\hline Total no. of nodes & 120 \\
Area & $1,000 \times 500$ \\
MAC & $802.15 .4,802.15 .6$ \\
No. of clusters & 15 \\
Simulation time & 200 seconds \\
Traffic source & CBR \\
Transmit power & 0.2 \\
Receiving power & 0.1 \\
Initial energy & 100 \\
No. of user nodes among 120 nodes & 10 \\
Storage device & 1 \\
Service provider & 6 \\
Task divider & 1 \\
Proxy server & 1 \\
Sensor nodes & 100 \\
\hline
\end{tabular}




\subsection{Performance evaluation metrics}

The performance of proposed protocol has been evaluated considering QoS metrics.

1 Delay: is the time difference between a time when the packet was sent from source node and time when packet arrives at the destination node.

2 Average residual energy consumption: energy consumed by sensor nodes for sensing, data communication and processing of data, calculated at the end of simulation is average residual energy consumption.

3 Throughput: the average number of packets that are successfully delivered to destination node per unit time is measured as throughput.

\subsection{Comparative graphs}

The simulation graph provides analysis of EESDRCB for being energy efficient and provides optimal routing by selectively turning off sensor node in each subcluster. TD provides separation of task based on data size and sends large data size information to be compressed by a proxy server and simultaneously assign tasks to the SP to reduce delay as shown in Figure 5 and average residual energy consumption as shown in Figure 6. Offloading the task of compressing increases throughput, with an increase in packet size as shown in Figure 7.

Figure 5 Delay vs. packet size using IEEE 802.15.4 and IEEE 802.15.6 (see online version for colours)

\section{Packet size Vs Delay}

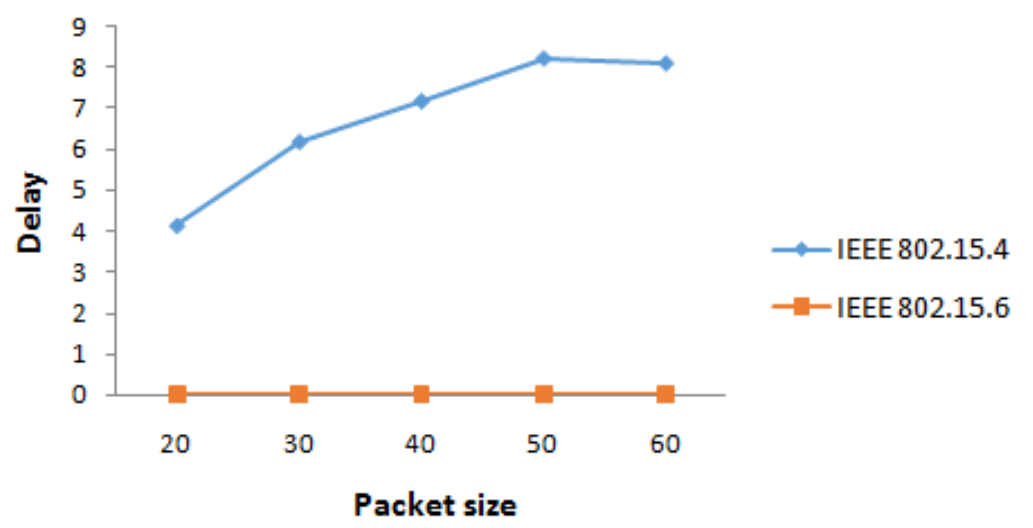

Figure 5 shows that by use of IEEE 802.15.6 results minimised delay compared to IEEE 802.15.4 since for the small amount of sensor data, compression is done at sensor node and forwarded immediately without delay. Large packets are compressed at the proxy with its high computing capability and sent to another SP. As a packet size increases, the transmission of large amounts of data from IEEE 802.15.4 incurs more delay than IEEE 802.15.6 since IEEE 802.15.6 has a higher data rate than IEEE 802.15.4. 
For a packet with size less than threshold, i.e., 50 bytes, compression of such packets carried at sensor node and for a packet with size greater than the threshold, compression done at the proxy server that has high computing capability. For packets with size greater than 50 bytes compression not done at sensor node. Thus, the delay has been minimised. While as packet size increases, time to compress packet increases which in turn increases delay till packet size 50 bytes.

Figure 6 depicts that average residual energy consumption with an increase in simulation time has decreased. Large size data are compressed at the proxy server and sensor nodes are concerned with only sensing and the data transmission task. Further, as the data transmission rate of IEEE 802.15.6 is high compared to IEEE 802.15.4, the number of sensor nodes can send a large amount of data without packet drop.

Figure 6 Average residual energy consumption with increase in simulation time (see online version for colours)

\section{Average Energy Consumption}

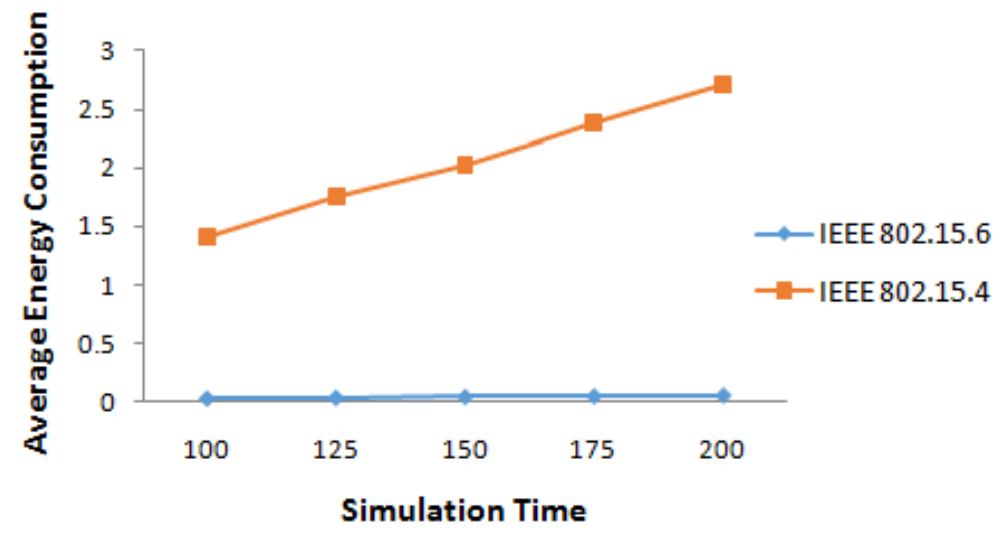

Figure 7 Throughput achieved using IEEE 802.15.4 and IEEE 802.15.6 (see online version for colours)

\section{Packet Size Vs Throughput}

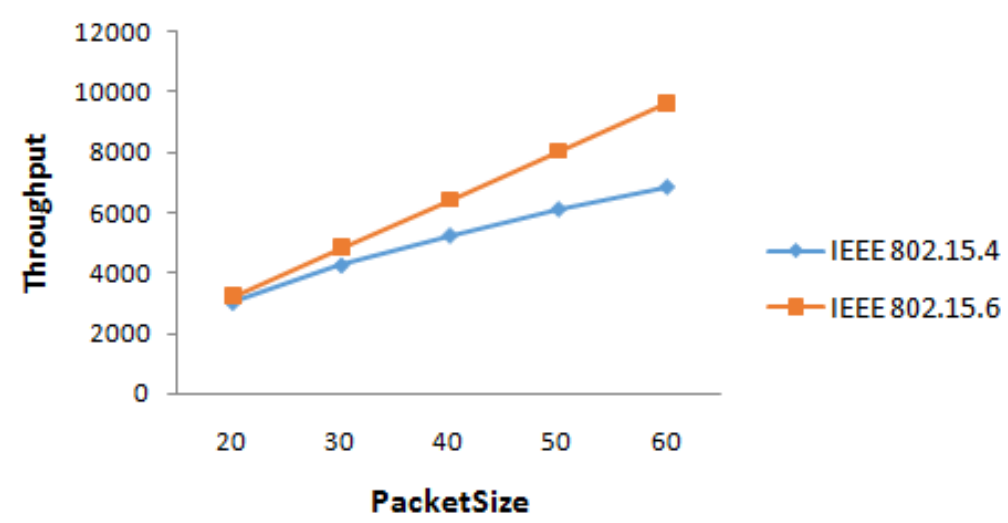


Figure 7 shows that by use of IEEE 802.15.6 and offloading the task of compressing large amounts of data to proxy server results in increased throughput with an increase in packet size. For a packet with size less than the set threshold, i.e., 50 bytes, compression of such packets carried at sensor node and for the packet with large size than the set threshold, compression done at the proxy server, which in turn, leads to increased throughput as TD assign small size compressed packet to the SP while a proxy server is performing compression of large size packet. The result also shows that throughput gained by use of IEEE 802.15.6 is more than IEEE 802.15.4 even with increased packet size as IEEE 802.15.6 has high data rate and can transfer a large amount of data between a proxy server and TD.

\section{Conclusions}

WSNs needs to achieve different QoS based on demands of several types of applications. Routing protocols for WSNs are designed to satisfy various QoS parameters to provide better performance and to increase the lifetime of the network. The use of cluster-based approach and stochastic scheduling algorithm highlights that design of EESDRCB using IEEE 802.15.6, or IEEE 802.15.4 achieves, various QoS metric like minimised energy consumption and delay compared to existing techniques in the literature. The use of differentiated service improves throughput with decreased delay.

\section{References}

Adamou, M., Lee, I. and Shin, I. (2001) An Energy Efficient Real-time Medium Access Control Protocol for Wireless Ad-hoc Networks, Report, YCS, Department of Computer Science, University of York, pp.5-8.

Agrakhed, J., Biradar, G.S. and Mytri, V.D. (2012) 'Adaptive multi constraint multipath routing protocol in wireless multimedia sensor network', in 2012 International Conference on Computing Sciences (ICCS), IEEE, September, pp.326-331.

Akkaya, K. and Younis, M. (2005) 'A survey on routing protocols for wireless sensor networks', Ad Hoc Networks, Vol. 3, No. 3, pp.325-349.

Alam, S., De, D. and Ray, A. (2015) 'Analysis of energy consumption for IARP, RIP and STAR routing protocols in wireless sensor networks', in 2015 Second International Conference on Advances in Computing and Communication Engineering (ICACCE), IEEE, May, pp.11-16.

Armbrust, M., Fox, A., Griffith, R., Joseph, A.D., Katz, R.H., Konwinski, A., Lee, G., Patterson, D.A., Rabkin, A., Stoica, I. and Zaharia, M. (2009) Above the Clouds: A Berkeley View of Cloud Computing, Technical Report UCB/EECS-2009-28, Vol. 4, pp.506-522, EECS Department, University of California, Berkeley.

Bai, X., Zhang, C., Xuan, D. and Jia, W. (2009) 'Full-coverage and k-connectivity $(k=14,6)$ three dimensional networks', in INFOCOM 2009, IEEE, IEEE, April, pp.388-396.

Elhoseny, M., Elleithy, K., Elminir, H., Yuan, X. and Riad, A. (2015) 'Dynamic clustering of heterogeneous wireless sensor networks using a genetic algorithm, towards balancing energy exhaustion', Int. J. Sci. Eng. Res., Vol. 6, No. 8, pp.1243-1252.

Elhoseny, M., Tharwat, A., Yuan, X. and Hassanien, A.E. (2018) 'Optimizing K-coverage of mobile WSNs', Expert Systems with Applications, Vol. 92, pp.142-153.

Heinzelman, W.B., Chandrakasan, A.P. and Balakrishnan, H. (2002) 'An application-specific protocol architecture for wireless microsensor networks', IEEE Transactions on Wireless Communications, Vol. 1, No. 4, pp.660-670. 
Jayashree, A., Biradar, G.S. and Mytri, V.D. (2012) 'Review of multipath routing protocols in wireless multimedia sensor network - a survey', International Journal of Scientific \& Engineering Research, Vol. 3, No. 7, pp.1-9.

Jian, C., Shaohua, Q., Yan, S. and Zenghong, H. (2014) Clustering Algorithm Based on Optimal Number of Clusters for Wireless Multimedia Sensor Networks, 077-5, ISBN: 978-1-84919928-5.

Jolly, G. and Younis, M. (2005) 'An energy-efficient, scalable and collision-free MAC layer protocol for wireless sensor networks', Wireless Communications and Mobile Computing, Vol. 5, No. 3, pp.285-304.

Kwak, K.S., Ullah, S. and Ullah, N. (2010) 'An overview of IEEE 802.15. 6 standard', in 2010 3rd International Symposium on Applied Sciences in Biomedical and Communication Technologies (ISABEL), IEEE, November, pp.1-6.

Lindsey, S. and Raghavendra, C.S. (2002) 'PEGASIS: power-efficient gathering in sensor information systems', in Aerospace Conference Proceedings, 2002, IEEE, Vol. 3, p.3.

Liu, H., Chandra, A. and Srivastava, J. (2005) dSENSE: Data-driven Stochastic Energy Management for Wireless Sensor Platforms, Tech. Rep. TR, pp.05-018, Dept. of CSE, Univ. of Minnesota.

Liu, H., Chandra, A. and Srivastava, J. (2006a) 'eSENSE: energy efficient stochastic sensing framework scheme for wireless sensor platforms', in Proceedings of the 5th International Conference on Information Processing in Sensor Networks, ACM, April, pp.235-242.

Liu, H., Chandra, A. and Srivastava, J. (2006b) PSS: Predictive Energy-Efficient Sensing Scheduling in Wireless Sensor Networks, Dept. of Computer Science, Minnesota Univ. Minneapolis.

Luo, H., Wang, J., Sun, Y., Ma, H. and Li, X.Y. (2010) 'Adaptive sampling and diversity reception in multi-hop wireless audio sensor networks', in 2010 IEEE 30th International Conference on Distributed Computing Systems (ICDCS), IEEE, June, pp.378-387.

Manjeshwar, A. and Agrawal, D.P. (2001) 'TEEN: a routing protocol for enhanced efficiency in wireless sensor networks', in Null, IEEE, April, p.30189a.

Manjeshwar, A. and Agrawal, D.P. (2002) 'APTEEN: a hybrid protocol for efficient routing and comprehensive information retrieval in wireless sensor networks', in IPDPS, IEEE, April, p.195b.

Patil, Y. and Agarkhed, J. (2015) 'A review on various issues and applications in wireless sensor networks', International Journal of Science and Research, Vol. 4, No. 11, pp.2518-2522.

Patil, Y. and Agarkhed, J. (2016) 'Offload computation in cloud assisted wireless sensor networks', in 2016 2nd International Conference on Advances in Electrical, Electronics, Information, Communication and Bio-Informatics (AEEICB), IEEE, February, pp.660-664.

Patil, Y.D., Agarkhed, J. and Patil, S.R. (2015) 'Error prediction scheduling for energy efficient routing in wireless sensor network', International Journal of Control Theory and Application, Vol. 8, No. 5, pp.1893-1902.

Shehab, A., Elhoseny, M. and Hassanien, A.E. (2018) 'An efficient scheme for video delivery in wireless networks', in Quantum Computing: An Environment for Intelligent Large Scale Real Application, pp.207-225, Springer, Cham.

Tjensvold, J.M. (2007) 'Comparison of the IEEE 802.11, 802.15. 1, 802.15. 4 and 802.15. 6 wireless standards', in IEEE, September, Vol. 18.

Wang, X., Xing, G., Zhang, Y., Lu, C., Pless, R. and Gill, C. (2003) 'Integrated coverage and connectivity configuration in wireless sensor networks', Proceedings of the 1st International Conference on Embedded Networked Sensor Systems, ACM, November, pp.28-39.

$\mathrm{Wu}, \mathrm{T}$. and Biswas, S. (2005) 'A self-reorganizing slot allocation protocol for multi-cluster sensor networks', in Proceedings of the 4th International Symposium on Information Processing in Sensor Networks, IEEE Press, April, p.41. 
Younis, M., Youssef, M. and Arisha, K. (2002) 'Energy-aware routing in cluster-based sensor networks', in 10th IEEE International Symposium on Modeling, Analysis and Simulation of Computer and Telecommunications Systems, 2002. MASCOTS 2002. Proceedings, IEEE, pp.129-136.

Younis, O. and Fahmy, S. (2004) 'Distributed clustering in ad-hoc sensor networks: a hybrid, energy-efficient approach', in Twenty-third Annual Joint Conference of the IEEE Computer and Communications Societies INFOCOM 2004, IEEE, March, Vol. 1.

Yu, J., Feng, L., Jia, L., Gu, X. and Yu, D. (2014) 'A local energy consumption prediction-based clustering protocol for wireless sensor networks', Sensors, Vol. 14, No. 12, pp.23017-23040.

Yuan, X., Elhoseny, M., El-Minir, H.K. and Riad, A.M. (2017) 'A genetic algorithm-based, dynamic clustering method towards improved WSN longevity', Journal of Network and Systems Management, Vol. 25, No. 1, pp.21-46.

Yun, Z., Bai, X., Xuan, D., Lai, T.H. and Jia, W. (2010) 'Optimal deployment patterns for full coverage and $\mathrm{k}$-connectivity $(\mathrm{k} \leq 6)$ wireless sensor networks', IEEE/ACM Transactions on Networking (TON), Vol. 18, No. 3, pp.934-947.

Zhang, Q., Fu, L., Gu, Y.J., Gu, L., Cao, Q., Chen, J. and He, T. (2014) 'Collaborative scheduling in dynamic environments using error inference', IEEE Transactions on Parallel and Distributed Systems, Vol. 25, No. 3, pp.591-601.

Zhang, Q., Gu, Y., He, T. and Sobelman, G.E. (2008) 'Cscan: a correlation-based scheduling algorithm for wireless sensor networks', in IEEE International Conference on Networking, Sensing and Control, 2008. ICNSC 2008, IEEE, April, pp.1025-1030.

Zhou, Z., Das, S. and Gupta, H. (2004) 'Connected k-coverage problem in sensor networks', in 13th International Conference on Computer Communications and Networks, 2004. ICCCN 2004. Proceedings, IEEE, October, pp.373-378. 